\title{
RICCI COLLINEATIONS FOR NON-DEGENERATE, DIAGONAL AND SPHERICALLY SYMMETRIC RICCI TENSORS.
}

\author{
G. Contreras*, \\ Laboratorio de Física Teórica, \\ Departamento de Física, Facultad de Ciencias, \\ Universidad de Los Andes, Mérida 5101, VENEZUELA \\ L.A. Núñez \\ Centro de Astrofísica Teórica, \\ Departamento de Física, Facultad de Ciencias, \\ Universidad de Los Andes, Mérida 5101, VENEZUELA \\ and Centro Nacional de Cálculo Científico \\ Universidad de Los Andes (CECALCULA), \\ Corporación Parque Tecnológico de Mérida, \\ Mérida 5101, VENEZUELA \\ U. Percoco $\ddagger$ \\ Centro de Astrofísica Teórica, \\ Departamento de Física, Facultad de Ciencias, \\ Universidad de Los Andes, Mérida 5101, VENEZUELA.
}

August 15, 2016

\begin{abstract}
The expression of the vector field generator of a Ricci Collineation for diagonal, spherically symmetric and non-degenerate Ricci tensors is obtained. The resulting expressions show that the time and radial
\end{abstract}

\footnotetext{
*In memoriam

${ }^{\dagger}$ nunez@ciens.ula.ve

${ }^{\ddagger}$ upercoco@ciens.ula.ve
} 
first derivatives of the components of the Ricci tensor can be used to classify the collineation, leading to 64 families.

Some examples illustrate how to obtain the collineation vector.

\section{Introduction.}

General Relativity provides a rich arena to use symmetries in order to simplify and understand the natural relation between geometry and matter furnished by the Einstein equations. Symmetries of geometrical/physical relevant quantities of this theory are known as Collineations and, in general, can be represented as: $£_{\vec{\xi}} \phi=F$, where $\phi$ and $F$ are two geometric objects, $\vec{\xi}$ is the vector field generating the symmetry and $£_{\vec{\xi}}$ the Lie Derivative along the congruence generated by $\vec{\xi}$. The hierarchy and relations among these symmetries are presented in the seminal work of Katzin and Levine [1] (see Fig.1 below). The particular position occupied by the Ricci Collineation (RC), defined by $\left(£_{\xi} R\right)_{a b}=0$, at the top of the hierarchical tree, and its close relation with the Energy-Momentum tensor, strongly motivate the study of Proper and Improper Ricci Collineations. Collineations can be proper or improper. A collineation of a given type is said to be proper if it does not belong to any of the subtypes. Clearly, in solving for example equation $\left(£_{\xi} R\right)_{a b}=0$, solutions representing improper collineations can be found. However, in order to be related to a particular conservation law, and its corresponding constant of the motion, the "properness" of the collineation type must be assured.

Ricci Collineations for static spherically symmetric space-times have been studied recently by various authors [2] [3] [- [4] [5] [6]. This work deals with the RC vector for dynamic (i.e. non-static) spherically symmetric space-times. A detailed analysis of the $\left(£_{\xi} R\right)_{a b}=0$ suggests a classification of $\mathrm{RC}$ based on the vanishing the time and radial first derivatives of the components of the Ricci tensor. This approach, as can be seen in the next section, leads to 64 families of $\mathrm{RC}$, each one distinguished by a set of vanishing first derivative of the components of the Ricci tensor; these results are summarized in Table 1.

Section three gives some examples of RC for some of the families listed in Table 1, with special emphasis on three specific FRW type metric tensors. 


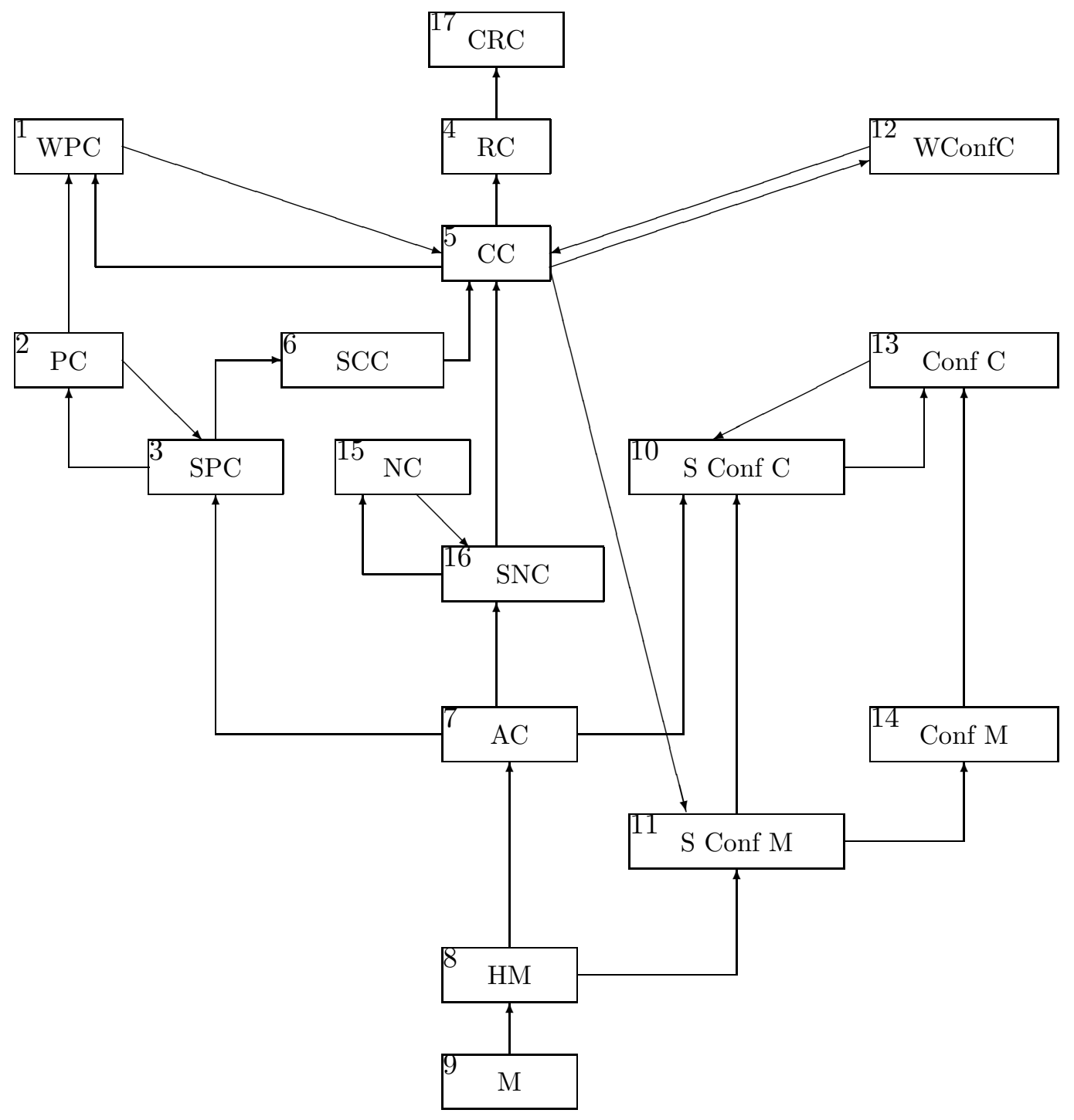

Fig.1. Relations between symmetries.

The leant arrows relate symmetries for which: $R_{i j}=0$.

1. WPC - Weyl Proyective Collineation: $£ W_{j k l}^{i}=0(n>2)$.

2. PC - Proyective Collineation: $£ \Gamma_{j k}^{i}=\delta_{j}^{i} \phi_{; k}+\delta_{k}^{i} \phi_{; j}$.

3. SPC - Special Proyective Collineation: $£ \Gamma_{j k}^{i}=\delta_{j}^{i} \phi_{; k}+\delta_{k}^{i} \phi_{; j}, \phi_{; j k}=0$.

4. RC - Ricci Collineation: $£ R_{i j}=0$.

5. CC - Curvature Collineation: $£ R_{j k l}^{i}=0$. 
6. SCC - Special Curvature Collineation: $\left(£ \Gamma_{j k}^{i}\right)_{; l}=0$.

7. AC - Affine Collineation: $£ \Gamma_{j k}^{i}=0$.

8. HM - Homothetic Motion: $£ g_{i j}=2 \sigma g_{i j}, \sigma=c t t e .$.

9. M - Motion: $£ g_{i j}=0$.

10. S Conf C - Special Conformal Collineation: $£ \Gamma_{j k}^{i}=\delta_{j}^{i} \sigma_{; k}+\delta_{k}^{i} \sigma_{; j}-g_{j k} g^{i l} \sigma_{; l}, \sigma_{; j k}=0$.

11. S Conf M - Special Conformal Motion: $£ g_{i j}=2 \sigma g_{i j}, \sigma_{; j k}=0$.

12. W Conf C - Weyl Conformal Collineation: $£ C_{j k l}^{i}=0,(n>3)$.

13. Conf C - Conformal Collineation: $£ \Gamma_{j k}^{i}=\delta_{j}^{i} \sigma_{; k}+\delta_{k}^{i} \sigma_{; j}-g_{j k} g^{i l} \sigma_{; l}$.

14. Conf M - Conformal Motion: $£ g_{i j}=2 \sigma g_{i j}$.

15. NC - null geodesic Collineation: $£ \Gamma_{j k}^{i}=g_{j k} g^{i m} \psi_{; m}$.

16. SNC - Special null geodesic Collineation: $£ \Gamma_{j k}^{i}=g_{j k} g^{i m} \psi_{; m}, \psi_{; j k}=0$.

17. CRC - Contracted Ricci Collineation: $g^{i j} £ R_{i j}=0$

\section{The expression of the $\mathrm{RC}$ vector field.}

Recently, the following result has been obtained by J. Carot et al. [7]: Therefore, the proper $R C$ of a spherically symmetric space-time whose Ricci tensor is non-degenerate, are of the form:

$$
\vec{\xi}=\xi^{t}(t, r) \partial_{t}+\xi^{r}(t, r) \partial_{r}
$$

Thus, we will integrate the system of equations $\left(£_{\vec{\xi}} R\right)_{a b}=0$ for a RC vector field with just two components ( $\xi^{t}$ and $\xi^{r}$ ) depending only of $t$ and $r$. Consideration of this collineation vector $\vec{\xi}$ does not preclude that another class of collineations, other than proper RC, could be obtained. Indeed: the form of the most general $\mathrm{RC}$ vector is the one given above plus linear combinations, with constant coefficients, of the Killing Vectors for spherical symmetry [7].

We consider a diagonal Ricci Tensor $R_{a b}$ written in those coordinates where ([8] pag.163 ):

$$
d s^{2}=-e^{2 \nu(t, r)} d t^{2}+e^{2 \lambda(t, r)} d r^{2}+Y^{2}(t, r)\left(d \theta^{2}+\operatorname{sen}^{2} \theta d \phi^{2}\right) .
$$

Then, the system of equations:

$$
C_{a b}=\left(£_{\vec{\xi}} R\right)_{a b}=0, \quad \text { with } \quad a, b=t, r, \theta, \phi
$$

reduces to:

$$
C_{t t}=\xi^{t} \partial_{t} A+\xi^{r} \partial_{r} A+2 A \xi^{t}{ }_{, t}=0
$$




$$
\begin{aligned}
C_{t r} & =A \xi_{, r}^{t}+B \xi^{r}{ }_{, t}=0 \\
C_{r r} & =\xi^{t} \partial_{t} B+\xi^{r} \partial_{r} B+2 B \xi_{, r}^{r}=0 \\
C_{\theta \theta} & =\xi^{t} \partial_{t} C+\xi^{r} \partial_{r} C=0
\end{aligned}
$$

where the following notation is used: $A=R_{t t}, B=R_{r r}$, and $C=R_{\theta \theta}$.

From the above equations (44) through (7)) we can get,

$$
\begin{aligned}
0 & =B C_{t t}+A C_{r r} \\
& =B \xi^{t} \partial_{t} A+B \xi^{r} \partial_{r} A+2 A B \partial_{t} \xi^{t}+A \xi^{t} \partial_{t} B+A \xi^{r} \partial_{r} B+2 A B \partial_{r} \xi^{r} \\
& =\left(B \partial_{t} A+A \partial_{t} B\right) \xi^{t}+\left(B \partial_{r} A+A \partial_{r} B\right) \xi^{r}+2 A B\left(\partial_{t} \xi^{t}+\partial_{r} \xi^{r}\right)
\end{aligned}
$$

Setting:

$$
\xi^{a}=(A B)^{-1 / 2} \eta^{a}
$$

with $A, B \neq 0$ and $\eta=\eta(t, r)$ we have,

$$
\partial_{a} \xi^{a}=-1 / 2(A B)^{-3 / 2}\left(B \partial_{a} A+A \partial_{a} B\right) \eta^{a}+(A B)^{-1 / 2} \partial_{a} \eta^{a}
$$

Now from equations.(8) and (10) it is clear that:

$$
2(A B)^{1 / 2} \partial_{a} \eta^{a}=0
$$

with the following solution:

$$
\eta^{a}=\epsilon^{a b} \partial_{b} \phi, \quad \phi=\phi(t, r)
$$

where, $\epsilon^{t r}=1, \epsilon^{r t}=-1$ and $\epsilon^{t t}=\epsilon^{r r}=0$

Let us now consider $C_{t r}$ :

$$
C_{t r}=A \partial_{r}\left[\eta^{t}(A B)^{-1 / 2}\right]+B \partial_{t}\left[\eta^{r}(A B)^{-1 / 2}\right]=0
$$

Differentiation with respect to $\phi$ and multiplying the result by $2(A B)^{3 / 2}$ we obtain:

$$
\left.C_{t r}=A\left(-\frac{\partial_{r}(A B)}{A B}\right) \partial_{r} \phi+2 \partial_{r r} \phi\right)+B\left(\frac{\partial_{t}(A B)}{A B} \partial_{t} \phi-2 \partial_{t t} \phi\right)=0
$$

From $C_{t t}, C_{\theta \theta}$ and $C_{t r}$ we have now the following system of equations:

$$
\begin{aligned}
A\left(-\frac{\partial_{r}(A B)}{A B} \partial_{r} \phi+2 \partial_{r r} \phi\right)+B\left(\frac{\partial_{t}(A B)}{A B} \partial_{t} \phi-2 \partial_{t t} \phi\right) & =0 \\
-\frac{\partial_{t} B}{B} \partial_{r} \phi-\frac{\partial_{r} A}{A} \partial_{t} \phi+2 \partial_{r t} \phi & =0 \\
\partial_{t} C \partial_{r} \phi-\partial_{r} C \partial_{t} \phi & =0
\end{aligned}
$$


and the form of the RC emerges as:

$$
\xi^{t}=\frac{\partial_{r} \phi}{\sqrt{A B}}, \quad \text { and } \quad \xi^{r}=-\frac{\partial_{t} \phi}{\sqrt{A B}} .
$$

From equations. (15), (16) and (17) we see that the partial derivatives of the components of the Ricci tensor will appear in the expression of $\vec{\xi}$. In order to classify all this sort of RC vectors, the afore mentioned equations suggest (almost obligate) to consider the vanishing of one or more derivatives of the components of the Ricci tensor as classifying parameters. With this criterion we get the 64 cases shown in TABLE 1 .

\section{Calculating a RC.}

In order to illustrate how to extract information from TABLE 1, we give some detailed examples.

\subsection{Family 1: $\partial_{r} A=0$.}

The equations (15), (16) and (17) take the following expression in this case:

$$
\begin{aligned}
\partial_{r} \phi \frac{\partial_{t} C}{\partial_{r} C}-\partial_{t} \phi & =0 \\
-\frac{\partial_{t} B}{B} \partial_{r} \phi+2 \partial_{t r} \phi & =0 \\
A\left(-\frac{\partial_{r}(B)}{B} \partial_{r} \phi+2 \partial_{r r} \phi\right)+B\left(\frac{\partial_{t}(A B)}{A B} \partial_{t} \phi-2 \partial_{t t} \phi\right) & =0 .
\end{aligned}
$$

From equations (20) and (21) we get:

$$
\partial_{r} \phi=\sqrt{B} f(r), \quad \text { and } \quad \partial_{t} \phi=\frac{\partial_{t} C}{\partial_{r} C} \sqrt{B} f(r) .
$$

Because $\partial_{r t} \phi=\partial_{r t} \phi$, we have

$$
f(r)=e^{\Delta^{(1)}}
$$

where

$$
\Delta^{(1)}=\frac{1}{2} \int \frac{B}{A} \frac{\partial_{t} C}{\partial_{r} C}\left(-\frac{\partial_{t}(A)}{A}+2 \frac{\partial_{t}\left(\frac{\partial_{t} C}{\partial_{r} C}\right)}{\frac{\partial_{t} C}{\partial_{r} C}}\right) d r
$$

Finally considering equations (18), the $\mathrm{RC}$ vector has the following components:

$$
{ }_{(1)} \xi^{t}=\frac{e^{\Delta^{(1)}}}{\sqrt{A}}, \quad \text { and } \quad{ }_{(1)} \xi^{r}=-\frac{\partial_{t} C}{\partial_{r} C} \frac{e^{\Delta^{(1)}}}{\sqrt{A}}
$$


3.2 Family 7: $\partial_{r} A=0$ and $\partial_{r} B=0$.

Equations (15), (16) and (17) take the following expression in this case:

$$
\begin{aligned}
\partial_{r} \phi \frac{\partial_{t} C}{\partial_{r} C}-\partial_{t} \phi & =0 \\
-\frac{\partial_{t} B}{B} \partial_{r} \phi+2 \partial_{t r} \phi & =0 \\
2 A \partial_{r r} \phi+B\left(\frac{\partial_{t}(A B)}{A B} \partial_{t} \phi-2 \partial_{t t} \phi\right) & =0
\end{aligned}
$$




\begin{tabular}{|c|c|c|c|}
\hline Family & vanishing derivatives & Family & vanishing derivatives \\
\hline 1 & $\partial_{r} A=0$ & 33 & $\partial_{t} A, \partial_{t} B, \partial_{r} B=0$ \\
\hline 2 & $\partial_{r} B=0$ & 34 & $\partial_{t} A, \partial_{t} B, \partial_{r} C=0$ \\
\hline 3 & $\partial_{r} C=0$ & 35 & $\partial_{t} A, \partial_{t} B, \partial_{t} C=0$ \\
\hline 4 & $\partial_{t} A=0$ & 36 & $\partial_{t} A, \partial_{t} C, \partial_{r} A=0$ \\
\hline 5 & $\partial_{t} B=0$ & 37 & $\partial_{t} A, \partial_{t} C, \partial_{r} B=0$ \\
\hline 6 & $\partial_{t} C=0$ & 38 & $\partial_{t} A, \partial_{t} C, \partial_{r} C=0$ \\
\hline 7 & $\partial_{r} A, \partial_{r} B=0$ & 39 & $\partial_{r} A, \partial_{t} B, \partial_{t} C=0$ \\
\hline 8 & $\partial_{r} A, \partial_{r} C=0$ & 40 & $\partial_{t} B, \partial_{t} C, \partial_{r} B=0$ \\
\hline 9 & $\partial_{r} B, \partial_{r} C=0$ & 41 & $\partial_{t} B, \partial_{t} C, \partial_{r} C=0$ \\
\hline 10 & $\partial_{t} A, \partial_{t} B=0$ & 42 & $\partial_{r} A, \partial_{t} A, \partial_{r} B, \partial_{r} C=0$ \\
\hline 11 & $\partial_{t} A, \partial_{t} C=0$ & 43 & $\partial_{r} A, \partial_{t} B, \partial_{r} B, \partial_{r} C=0$ \\
\hline 12 & $\partial_{t} B, \partial_{t} C=0$ & 44 & $\partial_{r} A, \partial_{r} B, \partial_{t} C, \partial_{r} C=0$ \\
\hline 13 & $\partial_{t} A, \partial_{r} A=0$ & 45 & $\partial_{r} A, \partial_{t} A, \partial_{r} B, \partial_{t} B=0$ \\
\hline 14 & $\partial_{r} A, \partial_{t} B=0$ & 46 & $\partial_{r} A, \partial_{t} A, \partial_{r} B, \partial_{t} C=0$ \\
\hline 15 & $\partial_{r} A, \partial_{t} C=0$ & 47 & $\partial_{r} A, \partial_{t} B, \partial_{r} B, \partial_{t} C=0$ \\
\hline 16 & $\partial_{t} A, \partial_{r} B=0$ & 48 & $\partial_{r} A, \partial_{t} A, \partial_{t} B, \partial_{r} C=0$ \\
\hline 17 & $\partial_{t} A, \partial_{r} C=0$ & 49 & $\partial_{r} A, \partial_{t} A, \partial_{t} C, \partial_{r} C=0$ \\
\hline 18 & $\partial_{t} B, \partial_{r} B=0$ & 50 & $\partial_{r} A, \partial_{t} B, \partial_{t} C, \partial_{r} C=0$ \\
\hline 19 & $\partial_{t} B, \partial_{r} C=0$ & 51 & $\partial_{t} A, \partial_{t} B, \partial_{r} B, \partial_{r} C=0$ \\
\hline 20 & $\partial_{t} C, \partial_{r} C=0$ & 52 & $\partial_{t} A, \partial_{r} B, \partial_{t} C, \partial_{r} C=0$ \\
\hline 21 & $\partial_{r} B, \partial_{t} C=0$ & 53 & $\partial_{t} B, \partial_{r} B, \partial_{t} C, \partial_{r} C=0$ \\
\hline 22 & $\partial_{r} A, \partial_{r} B, \partial_{r} C=0$ & 54 & $\partial_{t} A, \partial_{r} A, \partial_{t} B, \partial_{t} C=0$ \\
\hline 23 & $\partial_{r} A, \partial_{r} B, \partial_{t} A=0$ & 55 & $\partial_{t} A, \partial_{r} B, \partial_{t} B, \partial_{t} C=0$ \\
\hline 24 & $\partial_{r} A, \partial_{r} B, \partial_{t} B=0$ & 56 & $\partial_{t} A, \partial_{r} C, \partial_{t} B, \partial_{t} C=0$ \\
\hline 25 & $\partial_{r} A, \partial_{r} B, \partial_{t} C=0$ & 57 & $\partial_{t} A, \partial_{r} A, \partial_{t} B, \partial_{r} B, \partial_{r} C=0$ \\
\hline 26 & $\partial_{r} A, \partial_{t} A, \partial_{r} C=0$ & 58 & $\partial_{t} A, \partial_{r} A, \partial_{r} B, \partial_{t} C, \partial_{r} C=0$ \\
\hline 27 & $\partial_{r} A, \partial_{t} B, \partial_{r} C=0$ & 59 & $\partial_{r} A, \partial_{t} B, \partial_{r} B, \partial_{t} C, \partial_{r} C=0$ \\
\hline 28 & $\partial_{r} A, \partial_{t} C, \partial_{r} C=0$ & 60 & $\partial_{t} A, \partial_{r} A, \partial_{r} B, \partial_{t} C, \partial_{r} B=0$ \\
\hline 29 & $\partial_{t} A, \partial_{r} B, \partial_{r} C=0$ & 61 & $\partial_{t} A, \partial_{r} A, \partial_{t} B, \partial_{t} C, \partial_{r} C=0$ \\
\hline 30 & $\partial_{r} B, \partial_{t} B, \partial_{r} C=0$ & 62 & $\partial_{t} A, \partial_{t} B, \partial_{r} B, \partial_{t} C, \partial_{r} C=0$ \\
\hline 31 & $\partial_{r} B, \partial_{t} C, \partial_{r} C=0$ & 63 & $\partial_{t} A, \partial_{r} A, \partial_{r} B, \partial_{t} B, \partial_{t} C, \partial_{r} C=0$ \\
\hline 32 & $\partial_{r} A, \partial_{t} A, \partial_{t} B=0$ & 64 & $\partial_{t} A, \partial_{r} A, \partial_{r} B, \partial_{t} B, \partial_{t} C, \partial_{r} C \neq 0$ \\
\hline
\end{tabular}

TABLE 1: Families of RC vectors.

Again, from equations (27) and (28) we get:

$$
\partial_{r} \phi=\sqrt{B} f(r), \quad \text { and } \quad \partial_{t} \phi=\frac{\partial_{t} C}{\partial_{r} C} \sqrt{B} f(r)
$$


and $\partial_{r t} \phi=\partial_{r t} \phi$, yields,

$$
\frac{\partial_{r} f(r)}{f(r)}=-\frac{B}{2}\left(\frac{\partial_{t}(A B)}{A B} \frac{\partial_{t} C}{\partial_{r} C}-2 \partial_{t}\left(\frac{\partial_{t} C}{\partial_{r} C}\right)-\frac{\partial_{t} C}{\partial_{r} C} \frac{\partial_{t} B}{B}\right)
$$

so that,

$$
f(r)=e^{\Delta^{(7)}}
$$

where

$$
\Delta^{(7)}=\frac{B}{2} \int \frac{\partial_{t} C}{\partial_{r} C}\left(-\frac{\partial_{t}(A)}{A}+2 \frac{\partial_{t}\left(\frac{\partial_{t} C}{\partial_{r} C}\right)}{\frac{\partial_{t} C}{\partial_{r} C}}\right) d r=\frac{\Delta^{(1)}}{A}
$$

The components of the $\mathrm{RC}$ vector are:

$$
{ }_{(7)} \xi^{t}=\frac{e^{\Delta^{(7)}}}{\sqrt{A}}, \quad \text { and } \quad{ }_{(7)} \xi^{r}=-\frac{\partial_{t} C}{\partial_{r} C} \frac{e^{\Delta^{(7)}}}{\sqrt{A}}
$$

3.3 Family 64: $\partial_{c} R_{a b} \neq 0$ for $c=t, r$.

From equations (16), (17) and (18) we get,

$$
{ }_{(64)} \xi^{t}=\frac{e^{\Delta^{(64)}}}{\sqrt{A}}, \quad \text { and } \quad{ }_{(64)} \xi^{r}=\frac{-\partial_{t} C}{\partial_{r} C} \frac{e^{\Delta^{(64)}}}{\sqrt{A}}
$$

where

$$
\Delta^{(64)}=\frac{1}{2} \int \frac{\partial_{r} A}{A} \frac{\partial_{t} C}{\partial_{r} C} d t
$$

and the restriction equation emerging from equation.(15):

$$
A\left(-\frac{\partial_{r} A}{A}+2 \partial_{r} \Delta^{(64)}\right)+B \frac{\partial_{t} C}{\partial_{r} C}\left(\frac{\partial_{t} A}{A}-2 \frac{\partial_{t}\left(\frac{\partial_{t} C}{\partial_{r} C}\right)}{\frac{\partial_{t} C}{\partial_{r} C}}-\frac{\partial_{r} A}{A} \frac{\partial_{t} C}{\partial_{r} C}\right)=0
$$

with

$$
\partial_{r} C \neq 0
$$

\subsection{Three examples of FRW type metric tensors.}

\subsubsection{First Example:}

Consider the following line element [9],

$$
d s^{2}=d t^{2}-F^{2}(t)\left[\frac{1}{1-k r^{2}} d r^{2}+r^{2} d \theta^{2}+r^{2} \operatorname{sen}^{2} \theta d \phi^{2}\right]
$$


L. A. Núñez et al. have supposed a collineation vector of the following form:

$$
\vec{\xi}=\left(\xi^{t}(t, r), \xi^{r}(t, r), 0,0\right)
$$

The components of the Ricci tensor in this case are:

$$
A=-3 F_{, 00} / F, \quad B=\Delta /\left(1-k r^{2}\right), \quad C=r^{2} \Delta, \quad R_{33}=C \operatorname{sen}^{2} \theta
$$

where: $\Delta(t)=2 k+2\left(F_{, 0}\right)^{2}+F F_{, 00}$.

This metric tensor belongs to the family number $\mathbf{1}$ of the TABLE 1 $\left(\partial_{r} A=0\right)$. The components of the RC vector $\vec{\xi}$ are the following (see equations (25)):

$$
\begin{array}{r}
{ }_{(1)} \xi^{t}=\frac{w(t) \sqrt{1-k r^{2}}}{\sqrt{A}} \exp \left(-\frac{\partial_{t} \Delta}{4 k A}\left(-\frac{\partial_{t} A}{A}+2 \frac{\partial_{t}\left(\frac{\partial_{t} \Delta}{\Delta}\right)}{\left(\frac{\partial_{t} \Delta}{\Delta}\right)}\right)\right) \\
{ }_{(1)} \xi^{r}=-\frac{r \partial_{t} \Delta}{2 \Delta} \frac{w(t) \sqrt{1-k r^{2}}}{\sqrt{A}} \exp \left(-\frac{\partial_{t} \Delta}{4 k A}\left(-\frac{\partial_{t} A}{A}+2 \frac{\partial_{t}\left(\frac{\partial_{t} \Delta}{\Delta}\right)}{\left(\frac{\partial_{t} \Delta}{\Delta}\right)}\right)\right)
\end{array}
$$

Taking into account the equation (23), we can assume that

$$
w(t)=\text { const } \quad \text { and } \quad\left(-\frac{\partial_{t} \Delta}{4 k A}\left(-\frac{\partial_{t} A}{A}+2 \frac{\partial_{t}\left(\frac{\partial_{t} \Delta}{\Delta}\right)}{\left(\frac{\partial_{t} \Delta}{\Delta}\right)}\right)\right)=1
$$

Making it so, we obtain the $\mathrm{RC}$ vector in the same form that appears in the paper [9], and also the integrability condition (eq.2.13 of that paper).

\subsubsection{Second Example:}

In the paper of R. Chan et al. [10], for the case of null flux of heat, the following line element is considered:

$$
d s^{2}=-d t^{2}+B^{2}(t)\left[d r^{2}+r^{2}\left(d \theta^{2}+\sin ^{2} \theta d \phi^{2}\right)\right]
$$

where: $B(t)=\frac{M}{2 b} u^{2}$ and $u=\left(\frac{6 t}{M}\right)^{1 / 3}$.

This is a FRW metric with $k=0$. The Ricci Tensor is diagonal and its components are:

$$
R_{t t}=\frac{2}{3 t^{2}}, \quad R_{r r}=\frac{\left(6 M^{2}\right)^{1 / 3}}{b^{2} t^{2 / 3}}, \quad R_{\theta \theta}=R_{r r} r^{2}, \text { and } \quad R_{\phi \phi}=R_{\theta \theta} \sin ^{2} \theta
$$


In this case: $\partial_{r} A=0$ and $\partial_{r} B=0$. It belongs to the family number 7 of TABLE 1. Making use of equations (33), we obtain the following expressions for the components of the RC:

$$
{ }_{(7)} \xi^{t}=\sqrt{\frac{3}{2}} t \quad \text { and } \quad{ }_{(7)} \xi^{r}=\frac{r}{\sqrt{6}}
$$

This vector generates a Homothetic motion: $£_{\xi} g_{a b}=\alpha g_{a b}$ with $\alpha=\sqrt{\frac{3}{2}}$

\subsubsection{Third Example:}

Consider the metric [9]:

$$
d s^{2}=d t^{2}-R^{2}(t)\left(d r^{2}+r^{2} d \theta^{2}+r^{2} \sin ^{2} \theta d \phi^{2}\right)
$$

where: $R(t)=\beta t^{\alpha}, \alpha \neq 1$ and $\beta$ and $\alpha$ are constants.

This is also an example of a FRW metric whose Ricci tensor components are:

$$
\begin{aligned}
& R_{t t}=A=-3 \alpha(\alpha-1) t^{-2}, \quad R_{r r}=B=\beta^{2} t^{2 \alpha-2}\left(3 \alpha^{2}-\alpha\right), \\
& R_{\theta \theta}=C=\beta^{2} r^{2} t^{2 \alpha-2}\left[\alpha(\alpha-1)+2 \alpha^{2}\right], \quad R_{\phi \phi}=C \sin ^{2} \theta .
\end{aligned}
$$

Again, in this case $\partial_{r} A=0$ and $\partial_{r} B=0$, and it belongs to Family 7 of TABLE 1. From equations (33) we get the components of the RC vector:

$$
{ }_{(7)} \xi^{t}=c_{1} t, \quad \text { and } \quad{ }_{(7)} \xi^{r}=-c_{2} r
$$

where

$$
c_{1}=\frac{1}{\sqrt{-3 \alpha(\alpha-1)}} \quad \text { and } \quad c_{2}=-\frac{(\alpha-1)}{\sqrt{-3 \alpha(\alpha-1)}}
$$

are constants.

This vector generates a Homothetic motion: $£_{\xi} g_{a b}=g_{a b} \delta$, with $\delta=$ $\frac{1}{\sqrt{-3 \alpha(\alpha-1)}}$

\section{Final Comments.}

In this paper is presented the form of the Ricci Collineation Vector in the case of a diagonal, non-degenerate and spherically symmetric Ricci Tensor for space-times that would admit a proper RC. It also has been shown the way how to make the calculation of the $\mathrm{RC}$ for each one of the 64 families of Ricci tensors obtained. The way is open for the exploration of the nondiagonal and the degenerated Ricci tensors. 


\section{Acknowledgment.}

This work has been partially supported by Consejo Nacional de Investigaciones Científicas y Tecnológicas (CONICIT),Venezuela, under contract number 4179476 T-217-93.

The authors would also like to thank Dr. J. Carot for valuable suggestions.

\section{References}

[1] G.H. Katzin, J. Levine. J. Math. Phys. 10,(4),(1969).

[2] A.H. Bokhari, A. Qadir. J. Math. Phys. 34,(8),(1993).

[3] M.J. Amir, A.H. Bokhari, A. Qadir. J. Math. Phys.35,(6),(1994).

[4] T. Bin Farid, A. Qadir and M. Ziad. J. Math. Phys.36,(10),(1995).

[5] A.H. Bokhari and A.R. Kashif. J. Math. Phys.37,(7),(1996).

[6] R. Bertolotti, G. Contreras, L.A. Núñez, U. Percoco, J. Carot. J. Math. Phys. 37,(2), (1996).

[7] J. Carot, L.A. Núñez and U. Percoco. Gen. Rel and Grav.29,10,(1997).

[8] D. Kramer, H. Stephani, M. MacCallum. Exact Solutions of Einstein's Field Equations. Cambridge University Press(1980)

[9] L.A. Núñez, U. Percoco and V. Villalba. J. Math. Phys.31,1,(1990).

[10] R. Chan, J.P.S. Lemos, N.O. Santos, J.A. de F. Pacheco. Astroph. J.,342, 976,(1989) 